\title{
Morphologic Cross-Sectional Imaging Features of IgG4-Related Orbitopathy in Comparison to Ocular Adnexal Lymphoma
}

This article was published in the following Dove Press journal:

Clinical Ophthalmology

\author{
Annemarie Klingenstein (D) \\ Aylin Garip-Kuebler' \\ Siegfried Priglinger ${ }^{\prime}$ \\ Christoph Hintschich' \\ Ullrich G Mueller-Lisse ${ }^{2}$ \\ 'Department of Ophthalmology, Ludwig- \\ Maximilians-University, Munich, \\ Germany; ${ }^{2}$ Department of Radiology, \\ Ludwig-Maximilians-University, Munich, \\ Germany
}

\begin{abstract}
Aim: To detect radiological features that, in addition to clinical findings, may aid in correct differentiation between IgG4-related ophthalmic disease (IgG4-ROD) and ocular adnexal lymphoma (OAL).
\end{abstract}

Methods: In this retrospective, single-center, comparative analysis, we compared crosssectional imaging findings of 13 consecutive patients with histologically proven IgG4-ROD and a control group of 29 consecutive OAL-patients diagnosed between 10/2014 and 09/ 2019. Statistical significance was accepted at a $p<0.05$ significance level.

Results: IgG4-ROD-patients had longer time-to-diagnosis, higher orbital recurrence rates, but smaller lesions compared to OAL-patients $(p=0.002 ; p=0.006$ and $p=0.006$; Mann-Whitney $U$-test). Frequent cross-sectional imaging findings in both IgG4-RODpatients and OAL-patients included extraocular muscle enlargement (92\% and 93\%, respectively; most often in the lateral rectus muscles and the levator-complex), and lacrimal-gland enlargement ( $85 \%$ and $83 \%$, respectively). Other imaging findings comprised infraorbital nerve-involvement (IgG4-ROD, 23\%, OAL, 17\%) and orbital fat inflammation (IgG4-ROD, 23\%, OAL, 28\%). Bony infiltration and remodeling, heterogenous contrast-media distribution, and infiltration of the lacrimal system were seen slightly more often in IgG4-ROD $(23 \%, 38 \%, 15 \%$ and $15 \%$ versus $17 \%, 14 \%, 3 \%$ and $7 \%$ ). However, cross-sectional imaging features did not differ significantly between patient subgroups. Clinical symptoms predominantly occurred unilaterally (IgG4-ROD, 9/ 13, 69\%, OAL, 24/29, 83\%), while imaging findings were most often bilateral (IgG4ROD, 11/13, 85\%, OAL, 23/29, 79\%, $p<0.001$, McNemar test).

Conclusion: No morphological cross-sectional imaging sign could reliably distinguish between IgG4-ROD and OAL, leaving histopathology indispensable for definite diagnosis. Yet, importantly, for both IgG4-ROD and OAL, cross-sectional imaging frequently detected bilateral orbital disease when only one eye was clinically affected.

Keywords: IgG4, idiopathic orbital inflammation, CT, ocular adnexal lymphoma

\section{Introduction}

Recognition of IgG4-related orbital disease (IgG4-ROD) as a pathophysiological entity has reduced the group of patients left with idiopathic or nonspecific orbital inflammation. However, diagnosis of IgG4-ROD is often protracted ${ }^{1}$ and formal international treatment guidelines are still lacking. ${ }^{2}$ Radiological imaging features that could differentiate between IgG4-ROD and ocular adnexal lymphoma (OAL) as a common malignant differential diagnosis would be of great value.
Correspondence: Annemarie Kir

Department of Ophthalmology, Klinikum der Universität München, Campus Innenstadt, Mathildenstraße 8, Munich,

D-80336, Germany

Tel +4989440053811

Fax +4989440055160

Email Annemarie.Klingenstein@med.unimuenchen.de 
IgG4-ROD can be associated with sequential organ involvement ${ }^{3}$ in systemic IgG4-related disease, including lymphoplasmacytic organ infiltration with a high proportion of plasma cells expressing IgG4, and elevated IgG4 serum levels. ${ }^{4,5}$

Diagnostic criteria for IgG4-related systemic disease have been established ${ }^{6}$ and imaging studies associate IgG4-ROD with diffusely enlarged lacrimal glands, tendon-sparing extraocular muscle and infraorbital nerve enlargement, and inflammatory infiltration of the orbital fat. $^{7-12}$

Computed tomography (CT) imaging features of orbital lymphoid tumors include one or more lobulated or rounded orbital masses that mold to adjacent structures, and wedge-shaped enlargement of the lacrimal gland. ${ }^{13}$ However, inflammatory presentation is common for orbital lymphoid tumors, too, ${ }^{13}$ potentially impeding distinction from IgG4-ROD.

In the literature, the apparent diffusion coefficient (ADC) at diffusion-weighted MRI-imaging (DWI) has been significantly lower for lymphoma in comparison to orbital inflammation in general, ${ }^{14,15}$ and IgG4-ROD in particular, in limited patient collectives. ${ }^{16,17}$

Early recognition of IgG4-ROD and distinction from OAL is desirable for clinical management, especially in lesions that are small in size or in a posterior location that is risky for biopsy. Variable response to different immunosuppressive regimens has been observed in IgG4-ROD. As conversion from histologically proven IgG4-ROD to OAL is possible, ${ }^{1,18}$ repetitive biopsy may have to be considered. OAL-patients may benefit from extensive tumor staging, potentially involving combined wholebody positron-emission-/computed tomography (PET/ CT) ${ }^{19}$ In recent years, an increasing incidence of ocular non-Hodgkin-lymphomas has been reported. ${ }^{20}$

This retrospective, single-center, observational comparative study analyzed whether specific cross-sectional imaging features reliably distinguish between IgG4-ROD and OAL, such that they could aid in the differential diagnosis.

\section{Materials and Methods}

Institutional review board approval was obtained and individual patient consent waived by the ethics committee for this retrospective, single-center, observational comparative study of all patients identified by searching for the terms "OAL" and "IgG4-ROD" in the departmental histology database, and the procedure terms "Orbitotomy" and
"Orbital or conjunctival biopsy". The study is in adherence to the Declaration of Helsinki. Patients were histologically diagnosed with either IgG4-ROD $(n=13)$ or OAL $(n=29)$ between 10/2014 and 09/2019 and had cross-sectional imaging examinations performed prior to biopsy following written informed consent. Clinical data were completed from original patient files. All researchers had previously been involved with the clinical care of patients included.

Histopathological diagnosis represented the independent variable, with outcomes being either IgG4-ROD or OAL. Dependent variables, with potential outcomes of presence or absence, included clinical features of double vision, exophthalmos, globe dystopia, relative afferent pupillary defect (RAPD), eyelid edema/ptosis, and palpable orbital mass. Cross-sectional imaging features of extraocular muscle involvement (affecting the levator complex, lateral rectus muscle, medial rectus muscle, or inferior rectus muscle, respectively), lacrimal-gland enlargement, extraocular-tendon and extraocular-muscle enlargement, intraconal lesion, extraconal lesion (enlargement of extraocular muscle tendons or muscles accounted for both, extraconal and intraconal involvement), lesion in the respective upper or lower nasal or temporal orbital quadrant, heterogenous uptake of contrast media within the lesion, infiltration of the orbital fat body, infiltration of the lacrimal system, ethmoidal cell invasion, supraorbital nerve enlargement (implying infiltration), infraorbital nerve enlargement (implying infiltration), periorbital osseous remodeling, and periorbital osseous invasion were recorded separately for each eye.

Quantitative variables included respective lesion diameters in anterior-posterior (AP), lateral (LR), and craniocaudal (CC) dimensions of the largest visible lesion, and respective tumor volume estimate $(\mathrm{V})$ as calculated by the ellipsoid formula, $\mathrm{V}=\mathrm{AP} \times \mathrm{LR} \times \mathrm{CC} \times \pi / 6$.

Potential confounders included patient gender (female or male), age at diagnosis, time from clinical presentation to diagnosis, orbital recurrence of disease (presence or absence), previous diagnosis of systemic lymphoma (presence or absence), lesion laterality (right or left eye, both eyes), and best-corrected visual acuity (BCVA) [log(MAR)] of the symptomatic eye.

Ophthalmological examination included assessment of anamnesis (including onset and quality of symptoms), BCVA, slit-lamp examination, Goldmann-tonometry, Hertel-exophthalmometry, lid parameters, RAPD, diplopia, ophthalmometry in miosis and photo documentation. 
The cross-sectional imaging study closest in time to subsequent orbital biopsy (median time difference, 17 days) was retrieved for each patient from the institutional picture-archiving-and-communication system (PACS, Syngo, Siemens Healthineers, Erlangen, Germany) and displayed on 21-inch 5K-monitors licensed for medical image interpretation for consensus review in random order by one radiology attending with an interest in headand-neck radiology and 20 years of post-fellowship experience, and one ophthalmology attending specializing in oculoplastic surgery. Cross-sectional imaging findings were recorded separately for each orbit. Both observers were blinded to final histopathological diagnoses and had no access to other clinical or imaging information. Diagnostic multi-detector-row computed-tomography (CT, Optima 660 or Discovery 750, GE Healthcare) scans performed at $120 \mathrm{KVp}$ were reviewed in 37 patients, and magnetic-resonance imaging (MRI, Ingenia 3.0T or Ingenia S 1.5T, Philips Medical Systems) studies in five. Intravenous contrast media in standard doses were administered for imaging in the venous phase, approximately $60 \mathrm{~s}$ after commencing intravenous injection of contrast media, followed by normal saline solution, in all but one patient who reported previous severe reaction to intravenous contrast media and underwent unenhanced CT. Multi-planar imaging (MRI) or image-reformatting (CT) in the axial, coronal, and sagittal planes included small fields-of-view individually adapted to the morphological dimensions of the orbit, midface, and paranasal sinuses, with typical edge lengths of $12-16 \mathrm{~cm}$, and slice thicknesses between $0.6 \mathrm{~mm}$ and $3.0 \mathrm{~mm}$, respectively. MRI comprised T2-weighted, T1-weighted, and fat-suppressed T1-weighted imaging.

Histopathology findings were extracted from original reports. OALs were classified by TNM-staging as per the 7th Edition American Joint Committee on Cancer (AJCC) Staging Manual. ${ }^{21}$ Assessment was performed after formalin-fixation, paraffin embedding, sectioning and staining with hematoxylin and eosin as well as PAS (periodic acid-Schiff) staining. Additionally, immunohistochemistry assessed different markers (CD (=cluster of differentiation) 3, CD20, BCL (=B-cell-lymphoma) 2, BCL6, proliferation index Ki67, IgG, IgG4, Kappa and Lambda-CISH (chromogenic in situ hybridization) for IgG4-ROD and CD3, CD5, CD20, CD23, BCL2, BCL6 and Ki67 for OAL). IgG4-ROD was diagnosed based on established histopathological criteria (including, inter alia, IgG4/IgGratio $>40 \%$ and fibrosis) ${ }^{6}$
Data were tabulated in different Microsoft Excel worksheets for MacIntosh computers (Microsoft Corporation, Redmond, WA, USA, 2011). To avoid bias, respective cross-sectional imaging data, clinical data, and histopathology data were first recorded separately and combined for analysis only after completion of the data collection process.

Statistical analysis was performed with SPSS 25.0 (IBM Corporation; Armonk, NY, USA). Descriptive statistics, Mann-Whitney $U$-test, chi-square-test or Fisher's exact test, McNemar test and one-way analysis of variance (ANOVA) analyzed patients' clinical and functional data. Statistically, significance of differences was accepted for $p<0.05$ in two-tailed tests.

\section{Results}

Analysis was completed in all 42 patients, including 13 with histopathological IgG4-ROD (5 females), and 29 with OAL (14 females, no statistically significant difference, n.s.). Respective mean, median, and range of age at diagnosis of orbital disease were 58, 65, and 15-87 years for IgG4-ROD, and 65,65 , and 34-88 years for OAL (n.s.). BCVA of the symptomatic eye $[\log (\mathrm{MAR})]$ was 0.2 in IgG4-ROD and 0.3 in OAL patients (range $0-0.7$ and $-0.1-1.6$, respectively, $n$. s.). Time from onset of clinical symptoms to diagnosis was significantly longer in IgG4-ROD-patients (mean, median, range, 12, 6, 0-48 months) than in OAL-patients (7, 2, 1-48 months, $p=0.002$, Mann-Whitney $U$-test). Four IgG4-RODpatients $(31 \%)$, but no OAL-patient had recurrent orbital disease ( $p=0.006$, Mann-Whitney $U$-test).

Regarding ethnicity, one patient was Asian and one patient Arabian, both in the IgG4-ROD-patient group. All other patients were Caucasians.

One IgG4-ROD-patient had a prior diagnosis of IgG4associated pancreatitis and was under oral corticosteroid therapy, with elevated serum IgG4-levels preceding orbital manifestation by 15 months. The serum IgG4 level closest in time to orbital diagnosis was elevated at $11.8 \mathrm{~g} / 1$ (normal range $0.03-2.00 \mathrm{~g} / \mathrm{l}$ ). We could only find serum IgG4 levels drawn in one more patient at the time of orbital biopsy which was $0.496 \mathrm{~g} / 1$ (within the normal range).

IgG4-ROD patients were treated with systemic corticosteroids (prednisolone $1 \mathrm{mg} / \mathrm{kg}$ body weight) $(\mathrm{n}=10)$ for the most part, followed by intravenous rituximab therapy $(\mathrm{n}=2)$. One patient refused systemic therapy.

No OAL-patient had converted from IgG4-ROD. In seven OAL-patients (24\%), systemic lymphoma preceded orbital manifestation by a median of 56 months (range 4-156). 
Clinical TNM-staging was performed in all OAL-patients and distant metastasis as defined by the AJCC staging criteria was detected in eight OAL-patients (for details see Table 1). Lymphoma patients received a plethora of different therapy regimen throughout the course of their disease: We counted a total of radiation therapy in 12 , chemotherapy in 15 , immunomodulatory (anti-body) therapy in 5 and watchful waiting in 2 patients.

Orbital disease clinically affected one eye in nine and both eyes in four of the IgG4-ROD-patients, and one eye in 24 and both eyes in five of the OAL-patients (n.s.). Common orbital signs and symptoms at diagnosis included eyelid edema/ptosis (IgG4-ROD 8, 62\%, OAL 20, 69\%, n.s.), exophthalmos (IgG4-ROD 7, 54\%, OAL 10, 34\%, n.s.), and double vision (IgG4-ROD 6, 46\%, OAL 7, 24\%, n.s.). Less frequently, clinical examination revealed dystopia of the globe (IgG4ROD 2, 15\%, OAL 4, 31\%, n.s.), a palpable mass (IgG4ROD 1, 8\%, OAL 3, 10\%, n.s.), and RAPD (IgG4-ROD 0, OAL $1,3 \%$, n.s.).

Among cross-sectional imaging findings, lacrimal gland enlargement, extraocular muscle infiltration, involvement of supraorbital and infraorbital nerves, bony remodeling or invasion, heterogenous contrast uptake, orbital fat infiltration, extraconal versus intraconal lesions, localization in different orbital quadrants, infiltration of the lacrimal system, and ethmoidal cell invasion each did not differ significantly between IgG4-ROD and OAL (Mann-Whitney $U$-test). Respective percentages of imaging findings are listed both per orbit and per patient as well as p-values per patient and odds ratios in Table 2.

Extraocular muscle involvement was the most common radiological sign, being present in about $80 \%$ of orbits in both IgG4-ROD-patients and OAL-patients. It most frequently presented with peribulbar tendon enlargement at the zone of muscle attachment (Table 2). Figure 1 provides an example of circular periocular tendon infiltration of the lateral rectus muscles in a patient with lymphocytic OAL. The lateral rectus muscles showed the highest and the inferior rectus muscles the lowest rates of involvement (Table 2).

Supraorbital nerves were involved in about one-third, and infraorbital nerves in about one-fifth of each IgG4-RODpatients and OAL-patients, respectively (Table 2). Figure 2A and $\mathrm{B}$ show comparative examples of bilateral infiltration of infraorbital nerves on MRI and CT, respectively.
Table I Tumor Details in OAL Patients

\begin{tabular}{|c|c|c|c|}
\hline $\begin{array}{l}\text { Patient } \\
\text { No. }\end{array}$ & $\begin{array}{l}\text { TNM } \\
\text { Staging }\end{array}$ & $\begin{array}{l}\text { Distant } \\
\text { Metastasis }\end{array}$ & $\begin{array}{l}\text { Histopathological } \\
\text { Classification }\end{array}$ \\
\hline 1 & T2bNOMO & $n / a$ & MALT-lymphoma \\
\hline 2 & T2aNOMO & n/a & MALT-lymphoma \\
\hline 3 & T2cN3Mla & $\begin{array}{l}\text { Spleen, } \\
\text { intramuscular }\end{array}$ & Mantle cell lymphoma \\
\hline 4 & $\mathrm{~T} 2 \mathrm{bN} 4 \mathrm{M} 0$ & $n / a$ & ENMZL \\
\hline 5 & T2bN4MO & n/a & Mantle cell lymphoma \\
\hline 6 & T2aNOMO & $\mathrm{n} / \mathrm{a}$ & ENMZL \\
\hline 7 & T2aN4MO & n/a & $\begin{array}{l}\text { Non-Hodgkin B cell lymphoma } \\
\text { (not further classified) }\end{array}$ \\
\hline 8 & T3N4MO & $\mathrm{n} / \mathrm{a}$ & Follicular lymphoma \\
\hline 9 & $\mathrm{~T} 2 \mathrm{cN} 4 \mathrm{Mla}$ & $\begin{array}{l}\text { Subcutaneous tissue, } \\
\text { abdominal wall }\end{array}$ & Mantle cell lymphoma \\
\hline 10 & T3NOMO & $\mathrm{n} / \mathrm{a}$ & ENMZL \\
\hline 11 & T2cNOMO & n/a & ENMZL \\
\hline 12 & T2bNOMla & Cutaneous, breast & Follicular lymphoma \\
\hline 13 & T2bN3M0 & $\mathrm{n} / \mathrm{a}$ & ENMZL \\
\hline 14 & T2aNIMO & n/a & ENMZL \\
\hline 15 & T2NIMIa & Cerebral, breast & $\begin{array}{l}\text { Non-Hodgkin B cell lymphoma } \\
\text { (not further classified) }\end{array}$ \\
\hline 16 & $\mathrm{~T} 2 \mathrm{cN} 4 \mathrm{Mla}$ & $\begin{array}{l}\text { (Sub-)cutaneous, } \\
\text { kidney, liver, uterus }\end{array}$ & ENMZL \\
\hline 17 & T2cNOMO & n/a & ENMZL \\
\hline 18 & T4bN3M0 & n/a & Mantle cell lymphoma \\
\hline 19 & T2cNOMO & $\mathrm{n} / \mathrm{a}$ & ENMZL \\
\hline 20 & T2cNOMIb & Bone marrow & Lymphocytic lymphoma \\
\hline 21 & T2cNOMO & $\mathrm{n} / \mathrm{a}$ & ENMZL \\
\hline 22 & T2bNOMO & n/a & Follicular lymphoma \\
\hline 23 & T2bNIMO & $\mathrm{n} / \mathrm{a}$ & ENMZL \\
\hline 24 & T2bNOMla & Spleen & ENMZL \\
\hline 25 & T2bNOMO & $n / a$ & MALT-lymphoma \\
\hline 26 & T2cNOMO & $\mathrm{n} / \mathrm{a}$ & ENMZL \\
\hline 27 & T2bNIMO & n/a & ENMZL \\
\hline 28 & $\mathrm{~T} 2 \mathrm{bN} 4 \mathrm{Mla}$ & Cutaneous & Follicular lymphoma \\
\hline 29 & $\mathrm{~T} 2 \mathrm{cN} 3 \mathrm{MO}$ & $\mathrm{n} / \mathrm{a}$ & $\begin{array}{l}\text { Non-Hodgkin B cell lymphoma } \\
\text { (not further classified) }\end{array}$ \\
\hline
\end{tabular}

Abbreviations: MALT-lymphoma, mucosa-associated lymphoid tissue lymphoma; ENMZL, extranodal marginal zone lymphoma; $\mathrm{n} / \mathrm{a}$, not applicable. 
Table 2 Radiographic Findings in IgG4-ROD vs OAL Patients

\begin{tabular}{|c|c|c|c|c|c|c|}
\hline & $\begin{array}{l}\text { IgG4-ROD } \\
\text { n = Affected } \\
\text { Orbits }\end{array}$ & n = Patients & $\begin{array}{l}\text { OAL } \\
\text { n = Affected } \\
\text { Orbits }\end{array}$ & n = Patients & $\begin{array}{l}\text { p-value Mann- } \\
\text { Whitney U-test }\end{array}$ & $\begin{array}{l}\text { Odds } \\
\text { Ratio }\end{array}$ \\
\hline Extraocular muscle involvement & $20(77 \%)$ & 12 (92\%) & 47 (8I\%) & $27(93 \%)$ & 1.000 & 0.750 \\
\hline Levator complex & $15(58 \%)$ & $10(77 \%)$ & 37 (64\%) & $22(76 \%)$ & 1.000 & 0.865 \\
\hline Lateral rectus & $19(73 \%)$ & II (85\%) & $40(69 \%)$ & $24(83 \%)$ & 1.000 & 0.727 \\
\hline Medial rectus & $15(58 \%)$ & $8(62 \%)$ & $36(62 \%)$ & $19(66 \%)$ & 0.485 & 1.080 \\
\hline Inferior rectus & $14(54 \%)$ & $8(62 \%)$ & $32(55 \%)$ & $22(76 \%)$ & 1.000 & 1.719 \\
\hline Lacrimal gland enlargement & $16(62 \%)$ & II (85\%) & $37(64 \%)$ & $24(83 \%)$ & 1.000 & 0.727 \\
\hline Infraorbital nerve involvement & $6(23 \%)$ & $3(23 \%)$ & $9(16 \%)$ & $5(17 \%)$ & 0.485 & 0.582 \\
\hline Supraorbital nerve involvement & $5(19 \%)$ & $5(38 \%)$ & $5(9 \%)$ & $8(28 \%)$ & 0.681 & 0.667 \\
\hline Orbital fat infiltration & $4(15 \%)$ & $3(23 \%)$ & $10(17 \%)$ & $8(28 \%)$ & 1.000 & 1.212 \\
\hline Osseous infiltration & $4(15 \%)$ & $3(23 \%)$ & $5(9 \%)$ & $5(17 \%)$ & 0.681 & 0.667 \\
\hline Osseous molding & $7(27 \%)$ & $5(38 \%)$ & $6(10 \%)$ & $4(14 \%)$ & 0.102 & 0.246 \\
\hline $\begin{array}{l}\text { Inhomogenous contrast agent } \\
\text { distribution }\end{array}$ & $3(12 \%)$ & $2(15 \%)$ & I (2\%) & I (3\%) & 1.000 & 0.370 \\
\hline $\begin{array}{l}\text { Infiltration of the lacrimal system } \\
\text { and ethmoidal cells }\end{array}$ & $2(7 \%)$ & $2(15 \%)$ & $2(4 \%)$ & $2(7 \%)$ & 0.343 & 0.393 \\
\hline
\end{tabular}

Note: Odds ratio as per OAL in comparison to IgG4-ROD-patients.

Bilateral infiltration of the orbital fat is demonstrated in Figure 3 (patient with marginal cell OAL). The orbital fat was visibly altered in three (23\%) patients with IgG4-ROD and eight (28\%) patients with OAL.

Bony invasion and infiltration of the lacrimal system and ethmoidal cells was present in two patients in each

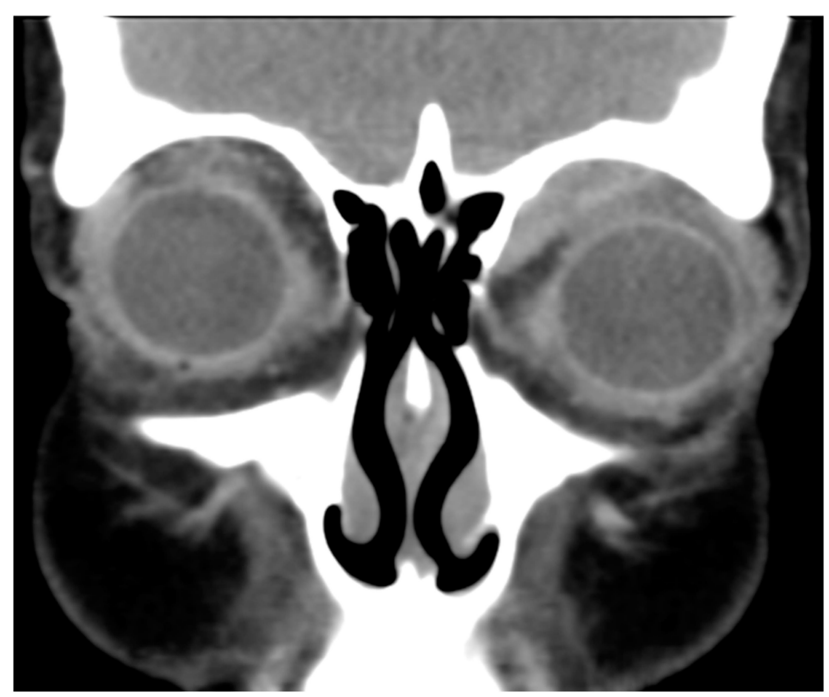

Figure I Bilateral periocular tendon involvement in a patient with OAL presenting via blurry muscle insertions of the four recti muscles (coronary CT with contrast agent, soft tissue window). group ( $15 \%$ of IgG4-ROD and $7 \%$ of OAL patients) and is shown in a patient with IgG4-ROD in Figure 4.

Based on V-estimates, tumor lesions were significantly smaller in IgG4-ROD-patients $\left(3.6 \pm 4.2 \mathrm{~cm}^{3}\right.$, mean $\left.\pm \mathrm{SD}\right)$ than in OAL-patients $\left(6.3 \pm 11.9 \mathrm{~cm}^{3}, p=0.006\right.$, Mann-Whitney $U$-test).

Five patients underwent MRI-examinations (IgG4 2, OAL 3 patients, respectively). However, diffusionweighted images (DWI) were obtained in only one patient who had his MRI-scan on site. The apparent-diffusion coefficient (ADC) of the right inferior IgG4-lesion was $1338 \pm 469 \mathrm{~mm} / \mathrm{s} 2$.

Except for differences in tumor lesion volume, no individual cross-sectional imaging feature distinguished between IgG4-ROD and OAL. When compared, however, clinical signs and symptoms were bilateral in 4/13 IgG4-RODpatients (31\%) and 5/29 OAL-patients (17\%), while crosssectional imaging findings involved both eyes more often, in 11/13 IgG4-ROD-patients (85\%) and 23/29 OAL-patients (79\%, $p<0.001$, McNemar-test). In consequence, bilateral cross-sectional imaging involvement was clinically inapparent in 7/13 IgG4-ROD-patients (54\%) and 18/29 OALpatients $(62 \%)$. When statistically comparing the number of patients presenting with bilateral disease between groups, no statistically significant difference could be found, neither 


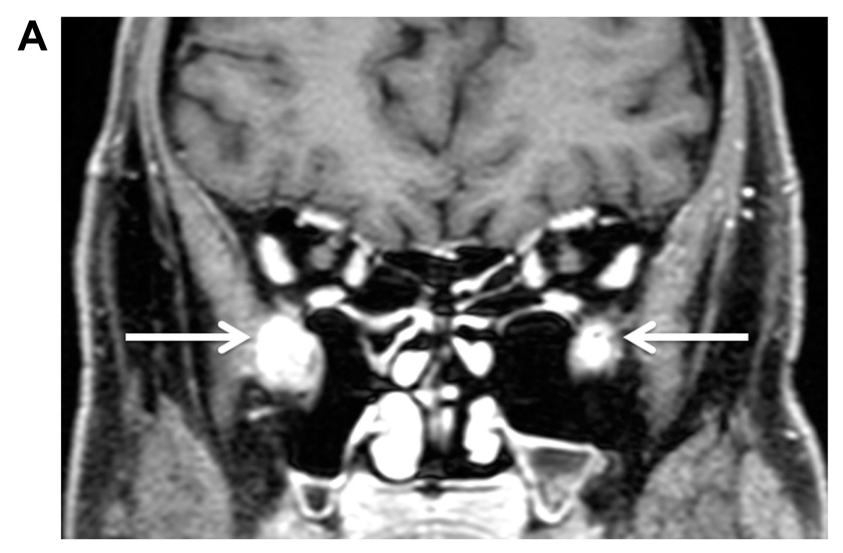

B

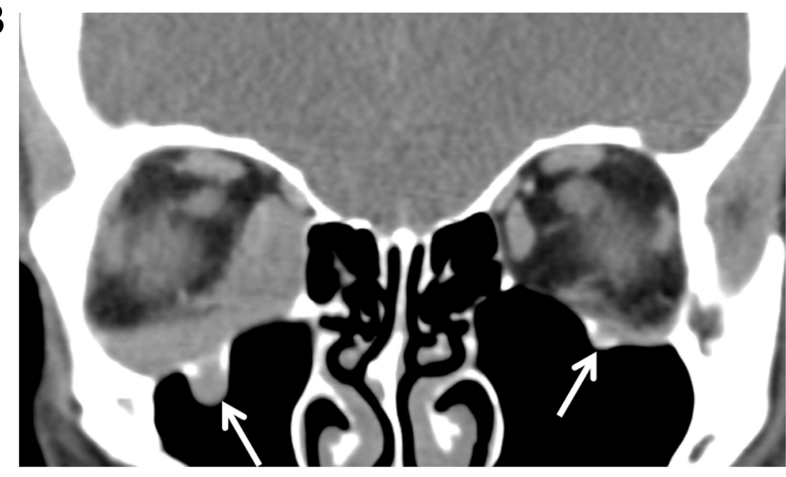

Figure 2 (A) Apparent bilateral infraorbital canal and nerve infiltration (arrows) in a patient with IgG4-ROD (coronary TI MRI scan with contrast agent). (B) In comparison bilateral infraorbital nerve infiltration (arrows) in a patient with mantle cell OAL (coronary CT scan with contrast agent, soft tissue window).

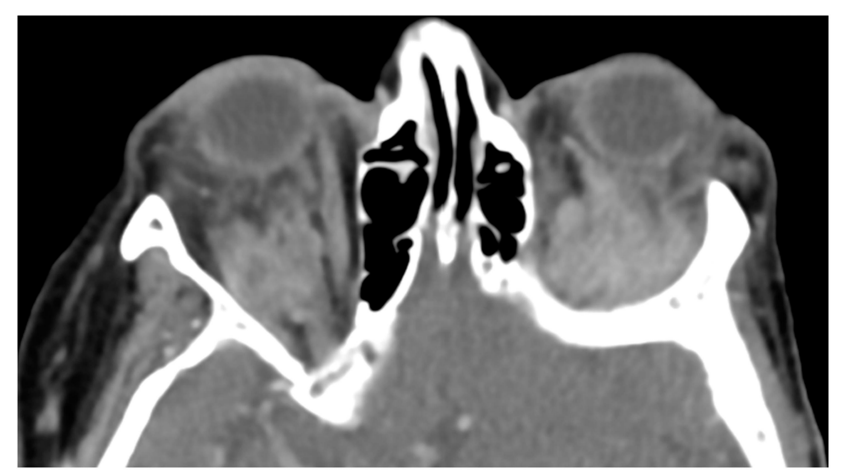

Figure 3 Bilateral infiltration of the orbital fat pad in a patient with OAL (axial CT scan with contrast agent, soft tissue window).

grouped for clinical nor radiographic bilateralism (MannWhitney $U$-test, n.s.).

\section{Discussion}

The most important results of this study include that, apparently, no specific clinical sign and no morphologic cross-sectional imaging sign reliably distinguishes between IgG4-ROD and OAL. Yet, time from clinical

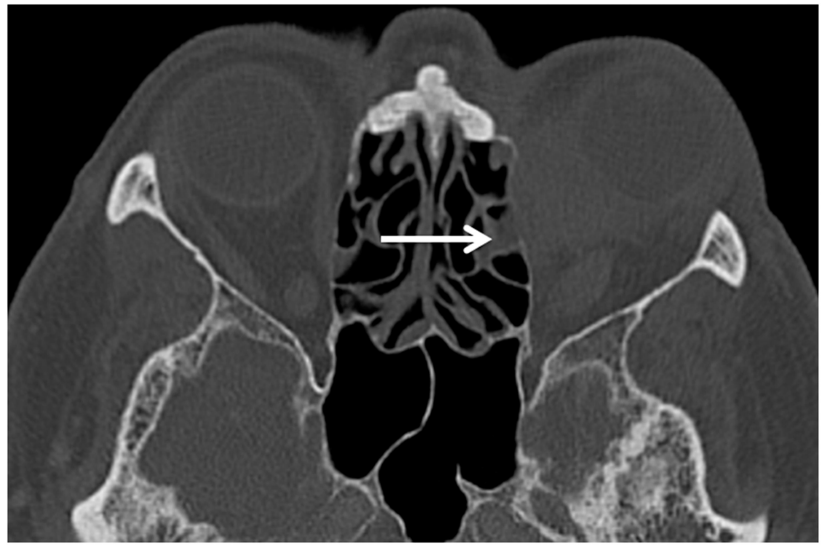

Figure 4 Infiltration of the lacrimal system and ethmoidal cells (arrow) in a patient with lgG4-ROD (axial CT scan with contrast agent, bone window).

onset of disease to diagnosis was significantly longer and tumorous lesions of IgG4-ROD were significantly smaller than in OAL. Importantly, cross-sectional imaging found bilateral orbital involvement significantly more often than clinical examination in both IgG4-ROD and OAL.

On radiological imaging, idiopathic orbital inflammation has been associated with dacryoadenitis, myositis, and apical, anterior, or diffuse disease. ${ }^{22}$ Cross-sectional imaging findings frequently associated with IgG4-ROD include extraocular muscle enlargement, lacrimal gland enlargement as well as enlargement of the infraorbital nerve and orbital fat. ${ }^{7-12}$

Like Tiegs-Heiden et al who examined 27 patients with IgG4-ROD ${ }^{7}$ we detected enlargement of the extraocular muscles in most patients with IgG4-ROD $\left(89 \%{ }^{7}\right.$ vs $92 \%$ in the present study). However, we also found extraocular muscle-enlargement in $93 \%$ of OAL-patients. The lateral rectus muscle was affected predominantly in both IgG4ROD and OAL, followed by the levator complex, in accordance with previous literature. ${ }^{7,22}$ Previous studies report sparing of the tendinous insertions of the extraocular muscles in IgG4-ROD, ${ }^{7,22}$ in contrast to orbital myositis which enlarges the entire muscle, including the tendon. ${ }^{22}$ Unlike previously reported, the present study frequently showed thickening of peribulbar tendons and adjacent blurriness of extraocular muscles, which were interpreted as signs of involvement with disease.

In a large imaging study evaluating orbital lymphoid tumors, intramuscular lymphoma has been described as rare and always associated with extramuscular masses. ${ }^{13}$ Multiple ocular adnexal lesions are found in many IgG4ROD-patients, too. ${ }^{23}$ Our results showed similar rates of extraocular muscle involvement and associated extramuscular masses in both IgG-ROD and OAL. 
On CT, orbital lymphoid tumors have been alternately described as lobulated, rounded masses that mold to adjacent structures, or as wedge-shaped masses filling the lacrimal gland. ${ }^{13}$ Lacrimal gland enlargement was the second most frequent cross-sectional imaging sign in the present study ( $85 \%$ of IgG4-ROD and $83 \%$ of OAL patients).

As previously reported, signs of both intraorbital inflammation and infraorbital nerve enlargement occurred in IgG4-ROD, ${ }^{7}$ but they accompanied OAL just as often.

Extraocular muscle enlargement and enlargement of the trigeminal nerve branch constitute risk factors for relapse in IgG4-ROD. ${ }^{23}$ Among our four relapsing IgG4ROD-patients, three (75\%) patients had extraocular muscle involvement and one (25\%) showed trigeminal nerve infiltration. It has been suggested that such lesions may confer refractoriness to systemic corticosteroid treatment, which has been recommended by consensus guidelines. ${ }^{23}$

Several publications have assessed DWI-MRI for distinction between benign and malignant orbital masses based on ADC values. ${ }^{14-17}$ Malignant masses, especially orbital lymphomas, have been associated with visually and quantitatively lower ADC-values as compared to benign masses. ${ }^{14-17}$ Among our patients, only five had MRI-examinations, and only one of those underwent DWI-imaging. The ADC-value of this patient's IgG4-ROD-lesion was within the range previously published. ${ }^{14}$ Yet, with histopathology remaining indispensable for correct diagnosis which is sustained by the results of the present study, routine implementation of DWI-MRI remains debatable due to limited accessibility on the one and extended costs on the other hand.

Of course, definite diagnosis should rely on histopathology, especially when considering possible malignancy. Yet, radiological criteria that correctly distinguish between benign and malignant orbital lesions would be of great value for challenging cases due to indistinct size or location of the lesion. Such criteria could justify repeated imaging in IgG4-ROD patients throughout the course of the disease and imply timing for re-biopsy, as these lesions could potentially convert to OAL. As there was no OAL patient in this study that had converted from IgG4, no specific sub-analysis could be performed in this regard.

Cross-sectional imaging lesions were present bilaterally in most patients, in contrast to clinical signs and symptoms that were mostly unilateral. Upon revision of patient records, we explain this by the subtle nature of some cross-sectional imaging findings, which may have been subclinical. We excluded interval disease progression between clinical and imaging examinations as a potential sort of bias, because time between clinical assessment, cross-sectional imaging, and surgery averaged 17 days. Thus, both eyes should be assessed very carefully when suspecting IgG4-ROD or OAL.

Inference from this study is limited in different ways. First, both IgG4-ROD and OAL represent rare disease entities, such that respective numbers of cases and comparative controls were rather limited despite the five-year recruitment-period at a large tertiary referral center. Second, for the same reasons, the study design was retrospective rather than prospective. Third, in attempts to assure similar quality levels and control for potential bias, only patients with histopathological proof of either IgG4-ROD or OAL and cross-sectional imaging prior to biopsy were included. However, in turn, this led to the inclusion of contrast-enhanced CT-scans in 36 patients, contrast-enhanced MRI in five, and unenhanced CT in one. In retrospect, DWI in MRI was performed in only one patient and thus ADC for possible differentiation of OAL from IgG4-ROD could thus only be calculated as an anecdotal finding in this single patient.

\section{Conclusions}

In conclusion, all cross-sectional imaging features reported for IgG4-ROD were also found in OAL patients in this study, with no statistically significant difference. It derives that orbital biopsy and histology remain indispensable for correct and timely diagnosis and initiation of therapy. Also, similarities at cross-sectional imaging impede the detection of conversion from IgG4-ROD to OAL. However, in both IgG4-ROD and OAL, cross-sectional imaging appears to detect significantly more eyes affected by disease than clinical examination alone. Longitudinal cohort studies appear warranted to follow the time-course of cross-sectional imaging key features.

\section{Abbreviations}

ADC, apparent diffusion coefficient; AJCC, American Joint Committee on Cancer; ANOVA, analysis of variance; AP, anterior-posterior; BCL, B-cell-lymphoma; BCVA, best corrected visual acuity; $\mathrm{CC}$, craniocaudal; $\mathrm{CD}$, cluster of differentiation; CISH, chromogenic in situ hybridization; CT, computed tomography; DWI, diffusion-weighted MRIimaging; ENMZL, extranodal marginal zone lymphoma; IgG4-ROD, IgG4-related ophthalmic disease; LR, lateral; MALT, mucosa-associated lymphoid tissue; MRI, magnetic resonance imaging; OAL, ocular adnexal lymphoma; PACS, picture-archiving-and-communication system; PAS, periodic 
acid-schiff; RAPD, relative afferent pupillary defect; V, tumor volume estimate.

\section{Data Sharing Statement}

The blinded datasets used and analyzed during the current study are available from the corresponding author upon reasonable request.

\section{Ethics Approval and Consent to Participate}

The study was waived by the ethics committee of Ludwig-Maximilians-University, Munich, Germany (project number 20-633 KB), giving their "declaration of no objection" and approving "no consulting obligation" regarding the retrospective evaluation of clinical and radiographic patient data of patients suffering from orbital lesions. All patients gave informed consent for crosssectional imaging examination. All patient data is nonidentifiable and complied with relevant data protection and privacy regulation.

\section{Consent for Publication}

Not applicable, no identifiable images included.

\section{Author Contributions}

All authors made substantial contributions to conception and design, acquisition of data, or analysis and interpretation of data; took part in drafting the article or revising it critically for important intellectual content; agreed to submit to the current journal; gave final approval of the version to be published; and agree to be accountable for all aspects of the work.

\section{Funding}

The authors did not receive support from any organization for the submitted work.

\section{Disclosure}

The authors report no conflicts of interest in this work.

\section{References}

1. Berry-Brincat A, Rose GE. Idiopathic orbital inflammation: a new dimension with the discovery of immunoglobulin G4-related disease. Curr Opin Ophthalmol. 2012;23(5):415-419. doi:10.1097/ICU.0b013e $32835563 \mathrm{ec}$

2. Detiger SE, Karim AF, Verdijk RM, et al. The treatment outcomes in IgG4-related orbital disease: a systematic review of the literature. Acta Ophthalmol. 2019;97(5):451-459. doi:10.1111/aos.14048
3. Khosroshahi A, Bloch DB, Deshpande V, et al. Rituximab therapy leads to rapid decline of serum IgG4 levels and prompt clinical improvement in IgG4 related systemic disease. Arthritis Rheum. 2010;62(6):1755-1762. doi:10.1002/art.27435

4. Carruthers MN, Stone JH, Khosroshahi A. The latest on IgG4-RD: a rapidly emerging disease. Curr Opin Rheumatol. 2012;24(1):60-69. doi:10.1097/BOR.0b013e32834ddb4a

5. Plaza J, Garrity J, Dogan A, et al. Orbital inflammation with IgG4-positive plasma cells; manifestations of IgG4 systemic disease. Arch Ophthalmol. 2011;129(4):421-428. doi:10.1001/archophthalmol. 2011.16

6. Okazaki K, Uchida K, Miyoshi H. Recent concepts of autoimmune pancreatitis and IgG4 related disease. Clin Rev Allergy Immunol. 2011;41(2):126-138. doi:10.1007/s12016-010-8214-2

7. Tiegs-Heiden $\mathrm{CA}$, Eckel LJ, Hunt $\mathrm{CH}$, et al. Immunoglobulin G4-related disease of the orbit: imaging features in 27 patients. AJNR Am J Neuroradiol. 2014;35(7):1393-1397. doi:10.3174/ajnr. A3865

8. Wallace ZS, Khosroshahi A, Jakobiec FA, et al. IgG4-related systemic disease as a cause of "idiopathic" orbital inflammation, including orbital myositis, and trigeminal nerve involvement. Surv Ophthalmol. 2012;57(1):26-33. doi:10.1016/j.survophthal.2011.07. 004

9. Katsura M, Mori H, Kunimatsu A, et al. Radiological features of IgG4-related disease in the head, neck, and brain. Neuroradiology. 2012;54(8):873-882. doi:10.1007/s00234-012-1012-1

10. Kubota T, Moritani S, Katayama M, et al. Ocular adnexal IgG4-related lymphoplasmacytic infiltrative disorder. Arch Ophthalmol. 2010;128 (5):577-584. doi:10.1001/archophthalmol.2010.45

11. Fujita A, Sakai O, Chapman M, et al. IgG4-related disease of the head and neck: CT and MR imaging manifestations. Radiographics. 2012;32(7):1945-1958. doi:10.1148/rg.327125032

12. Ginat DT, Freitag SK, Kieff D, et al. Radiographic patterns of orbital involvement in IgG4-related disease. Ophthal Plast Reconstr Surg. 2013;29(4):261-266. doi:10.1097/IOP.0b013e31829165ad

13. Polito E, Galieni P, Leccisotti A. Clinical and radiographical presentation of 95 orbital lymphoid tumors. Graefes Arch Clin Exp Ophthalmol. 1996;234(8):504-509. doi:10.1007/BF00184859

14. ElKhamary SM, Galindo-Ferreiro A, AlGhafri L, et al. Characterization of diffuse orbital mass using apparent diffusion coefficient in 3-tesla MRI. Eur J Radiol Open. 2018;5:52-57. doi:10.1016/j.ejro.2018.03.001

15. Jiang H, Wang S, Li Z, et al. Improving diagnostic performance of differentiating ocular adnexal lymphoma and idiopathic orbital inflammation using intravoxel incoherent motion diffusion-weighted MRI. Eur J Radiol. 2020;130:109191. doi:10.1016/j.ejrad.2020.109191

16. Hiwatashi A, Yoshiura T, Togao O, et al. Diffusivity of intraorbital lymphoma vs. IgG4-related DISEASE: 3D turbo field echo with diffusion-sensitised driven-equilibrium preparation technique. Eur Radiol. 2014;24(3):581-586. doi:10.1007/s00330-013-3058-9

17. Purohit BS, Vargas MI, Ailianou A, et al. Orbital tumours and tumour-like lesions: exploring the armamentarium of multiparametric imaging. Insights Imaging. 2016;7(1):43-68. doi:10.1007/s13244015-0443-8

18. Cheuk W, Yuen HK, Chan AC, et al. Ocular adnexal lymphoma associated with IgG4+ chronic sclerosing dacryoadenitis: a previously undescribed complication of IgG4-related sclerosing disease. Am J Surg Pathol. 2008;32(8):1159-1167. doi:10.1097/PAS. 0b013e31816148ad

19. Klingenstein A, Garip-Kuebler A, Mueller-Lisse UG, Hintschich C. Combined positron emission tomography/computed tomography for diagnosis and monitoring of orbital adnexal lymphoma. Acta Ophthalmol. 2018;96(6):e712-7. doi:10.1111/aos.13766

20. Moslehi R, Devesa SS, Schairer C, et al. Rapidly increasing incidence of ocular non-hodgkin lymphoma. J Natl Cancer Inst. 2006;98 (13):936-939. doi:10.1093/jnci/djj248 
21. Sniegowski MC, Roberts D, Bakhoum M, et al. Ocular adnexal lymphoma: validation of American Joint Committee on Cancer seventh edition staging guidelines. $\mathrm{Br} J$ Ophthalmol. 2014;98 (9):1255-1260. doi:10.1136/bjophthalmol-2013-304847

22. Ding ZX, Lip G, Chong V. Idiopathic orbital pseudotumour. Clin Radiol. 2011;66(9):886-892. doi:10.1016/j.crad.2011.03.018
23. Kubota T, Katayama M, Nishimura R, et al. Long-term outcomes of ocular adnexal lesions in IgG4-related ophthalmic disease. $\mathrm{Br}$ J Ophthalmol. 2020;104(3):345-349. doi:10.1136/bjophthalmol-201 8-313730

\section{Publish your work in this journal}

Clinical Ophthalmology is an international, peer-reviewed journal covering all subspecialties within ophthalmology. Key topics include: Optometry; Visual science; Pharmacology and drug therapy in eye diseases; Basic Sciences; Primary and Secondary eye care; Patient Safety and Quality of Care Improvements. This journal is indexed on PubMed

Submit your manuscript here: https://www.dovepress.com/clinical-ophthalmology-journal
Central and CAS, and is the official journal of The Society of Clinical Ophthalmology (SCO). The manuscript management system is completely online and includes a very quick and fair peer-review system, which is all easy to use. Visit http://www.dovepress.com/ testimonials.php to read real quotes from published authors. 